\title{
Private versus Social Incentives for Pharmaceutical Innovation*
}

\author{
Paula González† Inés Macho-Stadler; and David Pérez-Castrillo ${ }^{\ddagger}$
}

December 13, 2015

\begin{abstract}
We provide a theoretical framework to contribute to the current debate regarding the tendency of pharmaceutical companies to direct their R\&D toward marketing products that are "follow-on" drugs of already existing drugs, rather than toward the development of breakthrough drugs. We construct a model with a population of patients who can be treated with drugs that are horizontally and vertically differentiated. In addition to a pioneering drug, a new drug can be marketed as the result of an innovative process. We analyze physician prescription choices and the optimal pricing decision of an innovative firm. We also characterize the incentives of the innovative firm to conduct $R \& D$ activities, disentangling the quest for breakthrough drugs from the firm effort to develop follow-on drugs. Our results offer theoretical support for the conventional wisdom that pharmaceutical firms devote too many resources to conducting $\mathrm{R} \& \mathrm{D}$ activities that lead to incremental innovations.
\end{abstract}

Keywords: pharmaceuticals, R\&D activities, me-too drugs, breakthrough drugs, incremental innovation, radical innovation.

${ }^{*}$ We appreciate valuable comments by Bruno Jullien, Justina Klimaviciute, Jorge Mestre-Ferrándiz and participants at the XXIX Jornadas de Economía Industrial (Barcelona), the Workshop on Health Economics (UAB), the 16th European Health Economics Workshop (Toulouse), the XXXV AES Conference (Granada) and the 11th iHEA Conference (Milan). Financial support from Ministerio de Ciencia y Tecnología and FEDER (ECO2012-31962 and ECO2012-36480), Generalitat de Catalunya (2014SGR-142), Junta de Andalucía (SEJ-04992), ICREA Academia and the Severo Ochoa Programme (SEV-2011-0075) is gratefully acknowledged. Macho-Stadler and Pérez-Castrillo are fellows of MOVE and CESIfo.

${ }^{\dagger}$ Corresponding author. Department of Economics, Universidad Pablo de Olavide. Email: pgonzalez@upo.es

‡Department of Economics, Universitat Autònoma de Barcelona and Barcelona GSE. Email: Ines.Macho@uab.es

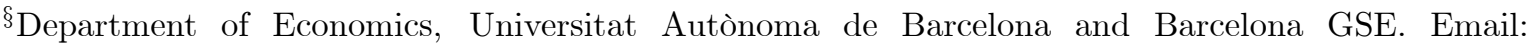
David.Perez@uab.es 


\section{Introduction}

It is widely recognized that of all the industrial sectors, the pharmaceutical industry is the sector that traditionally invests most heavily in research and development (R\&D). In 2012, for instance, US biopharmaceutical research companies invested an estimated $\$ 48.5$ billion in R\&D (PhRMA, 2013). Regarding R\&D intensity, and according to a recent report by the European Commission, spending on R\&D in 2012 by the pharmaceutical industry amounted to $15.3 \%$ of its GDP in the US, $16.3 \%$ in Japan, and to $14.7 \%$ in the European Union (European Commission, 2013).

However, there is a great deal of debate surrounding pharmaceutical R\&D activities. Pharmaceutical companies are often accused of devoting too many resources to the marketing of apparent new products that are "follow-on" drugs of already existing drugs, rather than toward the development of breakthrough (first-in-class) drugs. ${ }^{1}$ In fact, a successful new first-in-class drug will often face competition from a series of follow-on drugs that are therapeutically similar to the pioneering drug. The angiotensin converting enzyme (ACE) inhibitors, a class of drugs used to manage high blood pressure, is illustrative of this. The first ACE inhibitor, captopril, was introduced in the US in 1981. Since then over 10 ACE inhibitors have been launched (Hernandez and Harrington, 2008). ${ }^{2}$ The development of follow-on drugs is cheaper and less risky than drugs with a novel mechanism of action, but they supposedly do not bring significant therapeutic progress to patients (see, for instance, the discussions by Angell, 2004; Avorn, 2004; and Goozner, 2004). Defenders of incremental innovations argue, however, that medicines based on incremental improvements often represent advances in safety and efficacy, along with providing new formulations and dosing options that increase patient compliance (see diMasi and Paquette, 2004; Wertheimer and Santella, 2009; and the article by Doctor Henry I. Miller in the Wall Street Journal on January 1, 2014).

This paper aims at contributing to this social debate. We build a theoretical model of innovation to investigate whether there exist arguments that allow us to support the conviction that pharmaceutical firms devote too many resources to marketing me-too drugs and too few to launching breakthrough drugs. Our model emphasizes the distinction between radical and incremental innovation processes. ${ }^{3}$ Radical innovation processes may

\footnotetext{
${ }^{1}$ Follow-on drugs are sometimes called "me-too" drugs as they are close copies of existing drugs.

${ }^{2}$ Another example is omeprazole, the first proton pump inhibitor launched in 1989 to reduce gastric acid production. Proton pump inhibitors have since become the mainstay of treatment for acid-related gastrointestinal disease in adults, and omeprazole was followed by other proton pump inhibitors, with the most recent launched in 2009.

3 The labels "radical" and "incremental" belong mostly to the managerial literature and does not offer a unique description of the difference between the two concepts. In fact, the literature reveals that the definitions of radical and incremental innovations are still puzzling, both at the theoretical and at the
} 
lead to breakthrough drugs, while incremental innovation processes pursue me-too drugs.

In our model there is a continuum of patients in need of medical treatment. Patients can be treated with drugs that are horizontally and vertically differentiated. Vertical differentiation refers to the quality of the drug and includes the health gains experienced by patients. Horizontal differentiation reflects the adequacy of the drug for patients, as different patients in the population will experience different effects of a given medication in terms of tolerability, side effects or interaction with other medicines. In the market, there is a pioneering drug. We assume that the price of this drug is fixed, for example, because the patent protection that covered it has already expired and the drug is sold at its marginal cost. Moreover, a new drug can be marketed as the result of an innovative process by a pharmaceutical firm that seeks to achieve an improvement over the existing medicine. Finally, there is a physician who makes drug prescription decisions. The physician acts as a perfect agent for the health system (which includes both patients and the health authority) and, hence, he makes prescription choices based on the price-effectiveness of the drugs.

In this simple set-up we first describe physician prescription choices, given the prices and the characteristics of the two drugs (when the innovation process is successful). Second, we characterize the optimal pricing decision of the innovative firm, which anticipates the physician prescription behavior. The optimal price for the new drug depends on the differences in cost-effectiveness and the horizontal distance between the new drug and the pioneer. When the new drug is much more cost-effective than the pioneer, the innovative firm sets a price that leads the physician to prescribe the new drug to all patients. When the improvements in the cost-effectiveness of the new drug are not substantial, or the two drugs are very horizontally differentiated, then the price set by the innovative firm leads to a drug replacement treatment only for some patients. In all other situations the new drug is not marketed.

Finally, we characterize the incentives of the innovative firm to conduct $R \& D$ activities and compare these private incentives with those that would be optimal from a social point of view. The paper distinguishes between radical innovation processes, seeking breakthrough drugs, and incremental innovation processes that aim at launching a metoo drug. In order to differentiate these two kinds of innovations, we follow the approach of measuring the degree of innovativeness of a drug as the size of the differences (either small or large) between the new drug and the pioneer. These differences can emerge either in the horizontal or the vertical characteristics of the drugs. Innovations in the empirical level (see García and Calantone, 2002, for a critical review of the innovativeness terminology). In particular, the degree of innovativeness of a product is measured using various dimensions including the level of risk implied in the innovation strategy, the type of knowledge to be processed or the level of investment needed to move onto a new trajectory. 
vertical dimension imply a better quality of treatment (or a lower production cost) for all the patients suffering from the disease. ${ }^{4}$ Horizontal innovations would be advances that benefit some but not all patients because drugs may have lower side effects for a certain group of patients. ${ }^{5}$ Moreover, in order to account for the fact that the level of risk (or uncertainty of the final outcome) is typically larger in the case of radical innovations, we consider that the outcome of a radical innovation process by the innovative firm takes values on a large support and has a greater variance.

The paper provides some interesting findings. We show that for incremental innovation processes pursuing me-too drugs, the social value of the innovation coincides with the private benefits of the firm (as the innovative firm appropriates all the health system benefits derived from the launching of the me-too drug). If we consider, instead, $R \& D$ activities searching for breakthrough drugs, then private and social incentives for conducting research are not aligned. In particular, the incentives for conducting research by the firm are inferior to those socially optimal as there are patients that -despite the larger price of the new drug- benefit from it. These results allow us to show that if a pharmaceutical company can only adopt one of the two types of innovation processes due, for instance, to budget constraints, it may happen that the firm has an incentive to seek a me-too drug although $R \& D$ activities oriented to search for a radical innovation are socially superior. At the same time, it never happens that the innovative firm prefers to develop a radical innovation when devoting the resources to incremental innovations is preferable from a social point of view. Our results thus offer theoretical support for the conventional wisdom that pharmaceutical firms devote too many resources to conducting $\mathrm{R} \& \mathrm{D}$ activities that lead to me-too drugs.

The theoretical literature on incentives for pharmaceutical innovations is not abundant, although there is an increasing number of papers that study the interaction between the pricing policy constrained by various forms of regulation and the effort of innovation

\footnotetext{
${ }^{4}$ Examples of innovations that would be classified as vertical in our model would include the aforementioned captopril (ACE-inhibitor) and omeprazole (protone pump inhibitor), and also cimetidine (H2-receptor antagonist), propranolol (B-adrenoceptor antagonist), lovastatin (HMG-CoA-reductase inhibitor), and sumatriptane (5-HT1B/1D-receptor agonist) among others. All these are drugs that, when marketed, met a given need much more effectively than available treatments and were beneficial for all patients in the treatment of their disease. Also, innovations in antibiotics that allow administration once a day, giving patients the possibility of being treated at home, or at least the possibility to reduce hospitalization time, are vertical innovations according to our classification. Finally, second-generation antihistamines have some (vertical) improvements over first-generation antihistamines like, for instance, less frequent dosing.

${ }^{5}$ For example, in the market for statins, Lovastatin, pravastatin, and fluvastatin represent the class members with the lowest potency to reduce cholesterol levels but which are attractive candidates for use in treating patients who have proven intolerant of more potent statins such as atorvastatin, simvastatin or rosuvastatin (Kapur and Musunuru, 2008).
} 
by pharmaceutical firms. Ganuza, Llobet, and Domínguez (2009) find a bias in the pharmaceutical industry toward small innovations. Their result relies on the low sensitivity of a part of the demand (due to the loyalty of some physicians) to changes in prices. This lack of price-sensitivity provides an excessive reward for small innovations and consequently downwardly distorts the incentives of pharmaceutical firms. In our model, the physician acts as a perfect agent for the health system, so that the difference between the social value and the private benefits that the firm obtains from innovation arises from a different source: the ability of the pharmaceutical firm to appropriate or not the health system surplus through the price. The existence of physicians that are loyal to innovative drugs also plays an important role in Antoñanzas, Juárez-Castelló, and Rodríguez-Ibeas (2011). They study the incentives of an incumbent pharmaceutical firm to launch an upgraded drug through innovation before it faces generic competition. The paper shows that the equilibrium level of innovation exhibits an inverted $U$ shape, as innovation increases when the proportion of loyal physicians is low and decreases when it is high. Finally, Bardey, Bommier, and Jullien (2010) focus on the long-run impact of reference pricing on pharmaceutical innovation by firms. Their model shares some similarities with ours as it makes a clear distinction between incremental and radical innovations in a setting where drugs are horizontally and vertically differentiated. However, the distinction they make between the two types of innovations differs notably from ours. In addition to this, they model a patent race where the innovative process is deterministic and competition in $R \& D$ leads to the dissipation of firms' profits. They show that the short-term and long-term effects of price regulation may be antagonists. In their simulation using French data, they find that favoring radical innovation processes at the expense of cost-reducing innovations may generate medium/long-run increases in health expenses, despite potential short-run benefits.

The rest of the paper is organized as follows. The following section presents our model. Section 3 studies prescription decisions by the physician. Section 4 analyzes pricing decisions by the innovative firm and characterizes the market equilibrium. In section 5 we discuss the benefit to the health system derived from the launch of the new drug. Section 6 discusses private versus social incentives to innovate. Section 7 proposes a simple model of innovation. Section 8 discusses the robustnesss of the results when the physician acts as a perfect agent for the patient. Finally, the last section offers some concluding remarks. All the proofs are in the Appendix. 


\section{The Model}

There is a continuum of patients in need of medical treatment. We normalize the size of the population of patients to 1 . Patients suffer from the same illness and are identified by a horizontal characteristic $x$, with $x$ distributed uniformly on the interval $[0,1]$. The parameter $x$ represents the type of the patient and measures the heterogeneity regarding the patient genotype or any other characteristic that may induce the disease to have different effects among patients.

Patients can be treated with a drug. We consider that drugs are both horizontally and vertically differentiated. Thus, a drug is defined by a pair of characteristics: $(\hat{x}, \hat{h})$. The first characteristic $\hat{x} \in[0,1]$ captures the horizontal differentiation and reflects the adequacy of the drug for different patients. It is widely recognized that different patients in a population experience different effects of a given medication in terms of tolerability, side effects or interaction with other medicines. ${ }^{6}$ In our model, where patients are distributed along the interval $[0,1]$, a particular location (type) of a patient reflects the patient's ideal drug. That is, a drug with characteristic $\hat{x}$ has the ideal type for a patient located at $\hat{x}$. Those patients who fail to obtain a drug of their ideal type face a cost, and the farther patient type $x$ from $\hat{x}$ is, the lower the benefits he enjoys (or the larger the side effects he suffers) when he is exposed to the drug. ${ }^{7}$

The second characteristic of the drug incorporates the vertical differentiation and it refers to the gross effectiveness $\hat{h}$ of the drug. This is a quality dimension that affects the whole population and it includes the health gains experienced by patients (which may comprise either one or both quality and quantity of life). We assume $\hat{h}>0$, where $\hat{h}=0$ would mean that the drug has the same effect as no treatment, and the higher the $\hat{h}$ the better the drug for all the patients. ${ }^{8}$

In order to determine the health gain of a type- $x$ patient treated with drug $(\hat{x}, \hat{h})$, we need to consider both dimensions together. The health gain of a patient of type $x$ when $\operatorname{drug}(\hat{x}, \hat{h})$ is prescribed is $b(\hat{h}-l|\hat{x}-x|)$, where $|\hat{x}-x|$ is the distance between the

\footnotetext{
${ }^{6}$ For example, in the pharmaceutical market for blood pressure control, the drugs available to treat hypertension may act via the central nervous system, the heart (beta blockers), the kidney (diuretics, saluretics) or the vessels (alpha blockers, ACE inhibitors, AT1 and calcium antagonists). The efficacy and side effects of these medicines differ across patients and, hence, affect physician prescription patterns.

${ }^{7}$ There is no drug that unambiguously dominates another on the horizontal dimension, as patients with a different $x$ react differently to a drug $\hat{x}$. If two drugs with a different $\hat{x}$ are available, there are patients that benefit more from one of the drugs, while others would be better off when treated with the other.

${ }^{8}$ In Bardey et al. (2010) drugs are also vertically and horizontally differentiated, although their vertical characteristic is binary while ours is a continuous variable (which allows us to consider small and large differences in the vertical dimension).
} 
horizontal characteristic of the drug and the type of the patient, $l>0$ scales the loss of effectiveness or the extent of side effects and $b$ is the marginal utility of being healthy.

If we denote by $\hat{p}$ the price of drug $(\hat{x}, \hat{h})$, the benefit to the health system of treating patient $x$ with drug $(\hat{x}, \hat{h})$ and paying price $\hat{p}$ for this drug is

$$
H(x ; \hat{x}, \hat{h}, \hat{p})=b(\hat{h}-l|\hat{x}-x|)-\hat{p} .
$$

We assume that there is a pre-existing drug $\left(x^{0}, h^{0}\right), x^{0} \in(0,1)$, on the market. The price $p^{0}$ of this pioneering drug is exogenously fixed and does not react to the launch of a new medicine. In markets where drug $\left(x^{0}, h^{0}\right)$ is produced by a number of firms, this assumption can be easily justified on the grounds that the exclusivity of the pioneering drug has already expired and a generic drug has entered the market. ${ }^{9}$ Without loss of generality, we assume that $p^{0}=c^{0}$, being $c^{0}$ the marginal cost of providing the drug. For simplicity, we assume that all patients benefit from the pioneering drug, that is, the whole market is initially covered by that drug. ${ }^{10}$

There is a pharmaceutical firm (that we call firm 1), different from the one selling $\left(x^{0}, h^{0}\right)$, that can undertake an innovation process. ${ }^{11}$ This process is uncertain both because it may or may not lead to a new drug and because the characteristics of the potential new drug are ex-ante random. If firm 1 adopts an innovation process and the process is successful then a new drug is discovered. We denote by $\left(x^{1}, h^{1}\right)$ the characteristics of the new drug that will be marketed and by $c^{1}$ its marginal cost. This drug may use the same active principle as the pioneering drug or it may use a different one. What is relevant in our model is the location of the drug in the space of horizontal and vertical characteristics. We assume that the firm producing the new drug freely chooses the price $p^{1}$.

To identify the differences between the new drug and the pioneer, we define

$$
\begin{aligned}
\Delta_{x} & \equiv\left|x^{1}-x^{0}\right|, \\
\Delta_{h} & \equiv h^{1}-h^{0}, \\
\Delta_{c} & \equiv c^{1}-c^{0} .
\end{aligned}
$$

That is, $\Delta_{x} \in \mathbb{R}_{+}$is the distance between the "types" of the drugs. Similarly, $\Delta_{h} \in$ $\mathbb{R}$ indicates the difference in quality between the two drugs, and $\Delta_{c} \in \mathbb{R}$ denotes the

\footnotetext{
${ }^{9}$ In the US, the generic share of prescriptions rose from $19 \%$ in 1984 to $78 \%$ in 2010 (Berndt and Aitken, 2011). There is evidence that once generic drugs enter the market, both the price and sales revenue of pioneering drugs tend to drop by about $80 \%$ over the next year (Yin, 2012).

${ }^{10}$ This hypothesis requires that $b\left(h^{0}-l\left|x^{0}-x\right|\right)-c^{0} \geq 0$ for all $x \in[0,1]$, that is, $c^{0} \leq$ $\operatorname{Min}\left\{b\left(h^{0}-l\left(1-x^{0}\right)\right), b\left(h^{0}-l x^{0}\right)\right\}$.

${ }^{11}$ For simplicity in the exposition, we have adopted the view of an entrant in the pharmaceutical market launching a new drug. All our analysis would remain valid if, alternatively, we had assumed that there were several firms producing the pioneering drug and that one of them could undertake the innovation process, as in Ganuza et al. (2009).
} 
difference in marginal costs (the new drug may be more expensive or cheaper to produce than the pioneer).

Finally, there is a physician who makes drug prescription decisions. We assume that the physician acts as a perfect agent for the health system, which includes both the patients' utility and the cost incurred by the health authority. Hence, the physician assigns the medication to patients based on the price-effectiveness of the two drugs. ${ }^{12}$ Notice that, given the physician behavior, whether the patients bear a copayment or the drug is fully reimbursed by the health authority, there is no difference implied in the results since the physician takes into account the true cost of the drugs (paid either by the patients or the health authority). ${ }^{13}$

The timing of our game is as follows. In the first stage, firm 1 decides its innovation strategy. In case the innovation process succeeds, the characteristics $\left(x^{1}, h^{1}\right)$ as well as the marginal cost $c^{1}$ of the new drug are known. In the second stage, the innovative firm sets the price $p^{1}$ it charges. Finally, in the third stage, provided the new drug is marketed, the physician allocates the drugs to patients (if only the pioneering drug is available, the physician will prescribe it to all the patients). As usual, we solve the game by backward induction.

\section{Prescription of Drugs}

We now analyze the last stage of the game if a new drug with characteristics $\left(x^{1}, h^{1}\right)$ has been marketed at a price $p^{1}$. At this stage of the game, the physician decides which patients are prescribed the pioneering drug $\left(x^{0}, h^{0}\right)$ and which ones the new one $\left(x^{1}, h^{1}\right)$. The physician takes into account both the expected effectiveness of the drugs and their price. If both drugs provide identical benefits to the health system when treating a patient, we adopt the convention that the physician prescribes the new drug.

Following the physician's decision, the market will be split between the new drug and the pioneer. Depending on both drugs' characteristics, as well as the price $p^{1}$ decided by the innovative firm, three different scenarios may arise. We illustrate them in Figure 1.

First, the physician prescribes the pioneering drug to all patients if the price $p^{1}$ of the new drug is very high when compared to its health benefits. We denote $p^{\max }$ the price above which no patient is prescribed the new drug (that happens even when pa-

\footnotetext{
${ }^{12}$ This assumption allows us to focus on firm incentives to pharmaceutical innovation, leaving aside any distortion caused by the potential strategic behavior of agents as a consequence of the different views they share.

${ }^{13}$ In section 8 we discuss the robustness of the analysis in a model where the physician only takes into account the patients' utility. In that model, the reimbursement policy has an influence not only on physician prescription choices but also on the pricing and innovation decisions by the firm.
} 


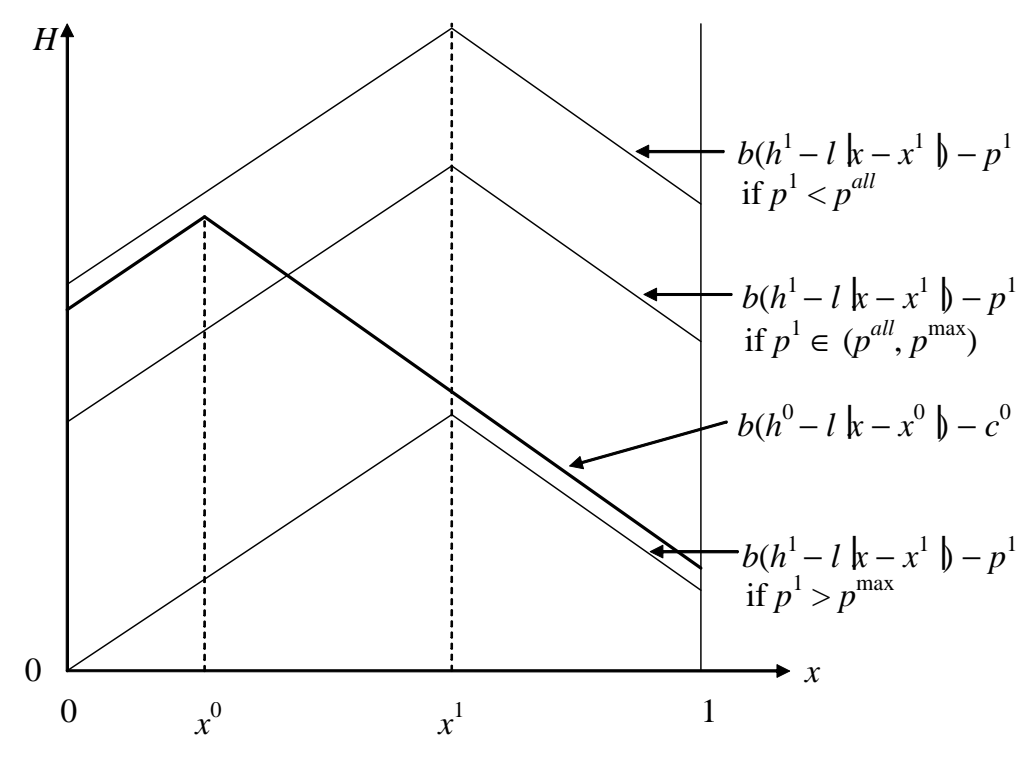

Figure 1: Benefits of the health system with the two drugs and split of the market as a function of $p^{1}, c^{0},\left(x^{0}, h^{0}\right)$, and $\left(x^{1}, h^{1}\right)$.

tient $x^{1}$ is not treated with $\left(x^{1}, h^{1}\right)$ if $\left.p^{1}>p^{\max }\right)$. Formally, $p^{\max }$ is characterized by $H\left(x^{1} ; x^{1}, h^{1}, p^{\max }\right)=H\left(x^{1} ; x^{0}, h^{0}, c^{0}\right)$, or $b\left(h^{1}-l\left|x^{1}-x^{1}\right|\right)-p^{\max }=b\left(h^{0}-l\left|x^{1}-x^{0}\right|\right)-$ $c^{0}$, which implies

$$
p^{\max } \equiv c^{0}+b\left(\Delta_{h}+l \Delta_{x}\right)
$$

If $p^{1}=p^{\max }$, the market for the new drug is $1-x^{1}$ if $x^{1}>x^{0}$; it is $x^{1}$ if $x^{1}<x^{0}$; and it is the whole market if $x^{1}=x^{0}$ (the rest of the patients are prescribed the old drug). Note that $p^{\max }$ is increasing in $b, l, \Delta_{x}, \Delta_{h}$, and $c^{0}$. Therefore, for a given $p^{1}$, it is more likely that the old drug will keep all the market if health has a low marginal value ( $b$ low), the disparities in the side effects of the drug for different patients are small ( $l$ low), the difference between the two drugs is also small ( $\Delta_{h}$ and $\Delta_{x}$ are low), and/or the pioneering drug is very cheap to produce.

Second, the physician prescribes the new drug to all patients if the price $p^{1}$ is low enough. This is the case if $p^{1}$ is so low that it is optimal to treat patient $x^{0}$ with the new drug. The cut-off value $p^{\text {all }}$ below which all patients are treated with $\left(x^{1}, h^{1}\right)$ is characterized by $b\left(h^{1}-l\left|x^{1}-x^{0}\right|\right)-p^{a l l}=b\left(h^{0}-l\left|x^{0}-x^{0}\right|\right)-c^{0}$, that is,

$$
p^{\text {all }} \equiv c^{0}+b\left(\Delta_{h}-l \Delta_{x}\right)
$$

Given the expression of $p^{\text {all }}$, a given $p^{1}$ is more likely to be lower than $p^{\text {all }}$ for large values of $c^{0}, b$, and $\Delta_{h}$, and for low values of $l$ and $\Delta_{x}$ (i.e., when the difference between 
the patient who profits more from each drug is low). We note that $p^{\text {all }}=p^{\max }$ if $x^{1}=x^{0}$.

Finally, the physician prescribes each drug to a subset of the patients for $p^{1} \in$ $\left(p^{\text {all }}, p^{\max }\right)$. To identify the market for each drug, consider first the case where $x^{1}>x^{0}$. The two drugs provide the same benefits to a patient of type $\tilde{x} \in\left(x^{0}, x^{1}\right)$ if $b\left(h^{0}-l\left(\tilde{x}-x^{0}\right)\right)-$ $c^{0}=b\left(h^{1}-l\left(x^{1}-\tilde{x}\right)\right)-p^{1}$. That is, the type of the indifferent patient is

$$
\tilde{x} \equiv x^{0}+\frac{\left(l \Delta_{x}-\Delta_{h}\right)}{2 l}+\frac{\left(p^{1}-c^{0}\right)}{2 b l} .
$$

The physician prescribes treatment $\left(x^{0}, h^{0}\right)$ at a price $c^{0}$ to those patients whose type lies in the interval $[0, \tilde{x})$, whereas patients with a type in the interval $[\tilde{x}, 1]$ are treated with $\left(x^{1}, h^{1}\right)$ at a price $p^{1}$.

Similarly, for $p^{1} \in\left(p^{\text {all }}, p^{\max }\right)$ and $x^{1}<x^{0}$ the indifferent patient is

$$
\tilde{x} \equiv x^{0}-\frac{\left(l \Delta_{x}-\Delta_{h}\right)}{2 l}-\frac{\left(p^{1}-c^{0}\right)}{2 b l}
$$

and the markets for the new drug and the pioneer are $[0, \tilde{x}]$ and $(\tilde{x}, 1]$, respectively.

\section{The Optimal Pricing Policy}

In this section we study the optimal price decision of firm 1 if, as a result of the innovation process, a new drug with characteristics $\left(x^{1}, h^{1}\right)$ has been achieved.

Firm 1's incentive to price high or low depends on the drug differences in terms of vertical characteristics (both in quality, measured by $\Delta_{h}$, and in marginal cost, measured by $\Delta_{c}$ ), and horizontal characteristics (measured by the distance in type $\Delta_{x}$ ). The strength of the incentives also depends on the size of the submarket (the subset of patients) in which the innovative firm more directly competes with the pioneering drug. If $x^{1}$ is on the right of $x^{0}$ (i.e., $x^{1}>x^{0}$ ), then the competition is fiercer on the right of $x^{0}$ than on the left of $x^{0}$. That is, the size of the submarket in which firm 1 directly challenges the pioneering drug is $1-x^{0}$. Similarly, if $x^{1}<x^{0}$, the competition is more intense to the left of $x^{0}$, that is, in a submarket of size $x^{0}$. We denote by $M$ the size of this submarket: $M \equiv 1-x^{0}$ if $x^{1}>x^{0}$ and $M \equiv x^{0}$ if $x^{1}<x^{0}$. We also denote $M \equiv 1$ if $x^{1}=x^{0}$ (although $M$ does not play any role when $\left.\Delta_{x}=0\right)$.

Proposition 1 summarizes the price decision of the pharmaceutical firm. Except for the region of parameters where there is no room for the firm to make profits, the new drug is always offered at a price in the interval $\left[p^{\text {all }}, p^{\max }\right]$. For convenience, we define

$$
p^{i n t} \equiv \frac{1}{2}\left(c^{0}+c^{1}\right)+b l M-\frac{1}{2} b\left(l \Delta_{x}-\Delta_{h}\right),
$$

the candidate price for an interior solution. 
In Proposition 1, we also use the function $g\left(\Delta_{x}\right)$, which is defined as follows:

$$
\begin{aligned}
g\left(\Delta_{x}\right) & \equiv \Delta_{x}+2(2-M-2 \sqrt{1-M}) \quad \text { if } \Delta_{x}>\sqrt{1-M}-(1-M) \\
& \equiv \frac{\left(1+M-\Delta_{x}\right)}{\left(1-M+\Delta_{x}\right)} \Delta_{x} \quad \text { otherwise. }
\end{aligned}
$$

The function $g\left(\Delta_{x}\right)$ is increasing, continuously differentiable and fulfills $g\left(\Delta_{x}=0\right)=0$.

Finally, as $\Delta_{h}$ and $\Delta_{c}$ have similar effects and as they often appear together in the mathematical expressions, we denote the composite effect of these two vertical variables as

$$
\Delta_{y} \equiv \Delta_{h}-\frac{1}{b} \Delta_{c}
$$

The parameter $\Delta_{y}$ can be interpreted as a measure of the differences in cost-effectiveness between the new drug and the pioneering one. The larger the value of $\Delta_{y}$ the more cost-effective the new drug is compared to the pioneer.

Proposition 1 The optimal price decision $p^{1 *}$ of firm 1 and its profits $\Pi^{1}\left(p^{1 *}\right)$ are as follows:

Region a: If $\Delta_{y} \leq-l \Delta_{x}$ then the new drug is not prescribed: $p^{1 *}=c^{1}$ and $\Pi^{1}\left(c^{1}\right)=0$.

Region b: If $\Delta_{y} \in\left(-l \Delta_{x}, \lg \left(\Delta_{x}\right)\right)$ then the new drug replaces the pioneer for a subset of patients. If in addition

Region b.i: $M \geq \frac{1}{2 l}\left(\Delta_{y}+3 l \Delta_{x}\right)$ then $p^{1 *}=p^{\max }$ and

$$
\Pi^{1}\left(p^{\max }\right)=b\left(M-\Delta_{x}\right)\left(\Delta_{y}+l \Delta_{x}\right) .
$$

Region b.ii: $M<\frac{1}{2 l}\left(\Delta_{y}+3 l \Delta_{x}\right)$ then $p^{1 *}=p^{\text {int }}$ and

$$
\Pi^{1}\left(p^{i n t}\right)=\frac{b}{2 l}\left(l M+\frac{1}{2}\left(\Delta_{y}-l \Delta_{x}\right)\right)^{2} .
$$

Region c: If $\Delta_{y} \geq \lg \left(\Delta_{x}\right)$ then the new drug takes over the entire market: $p^{1 *}=p^{\text {all }}$, and

$$
\Pi^{1}\left(p^{\text {all }}\right)=b\left(\Delta_{y}-l \Delta_{x}\right)
$$

The optimal profit function $\Pi^{1}$ is continuous in $\Delta_{x}, \Delta_{h}$, and $\Delta_{c}$.

We now offer some intuitions for Proposition 1. When differences in the cost-effectiveness between the new drug and the pioneer are very negative, that is, for low values of $\Delta_{y}$, there is no price above the marginal cost under which firm 1 can sell its drug. More 


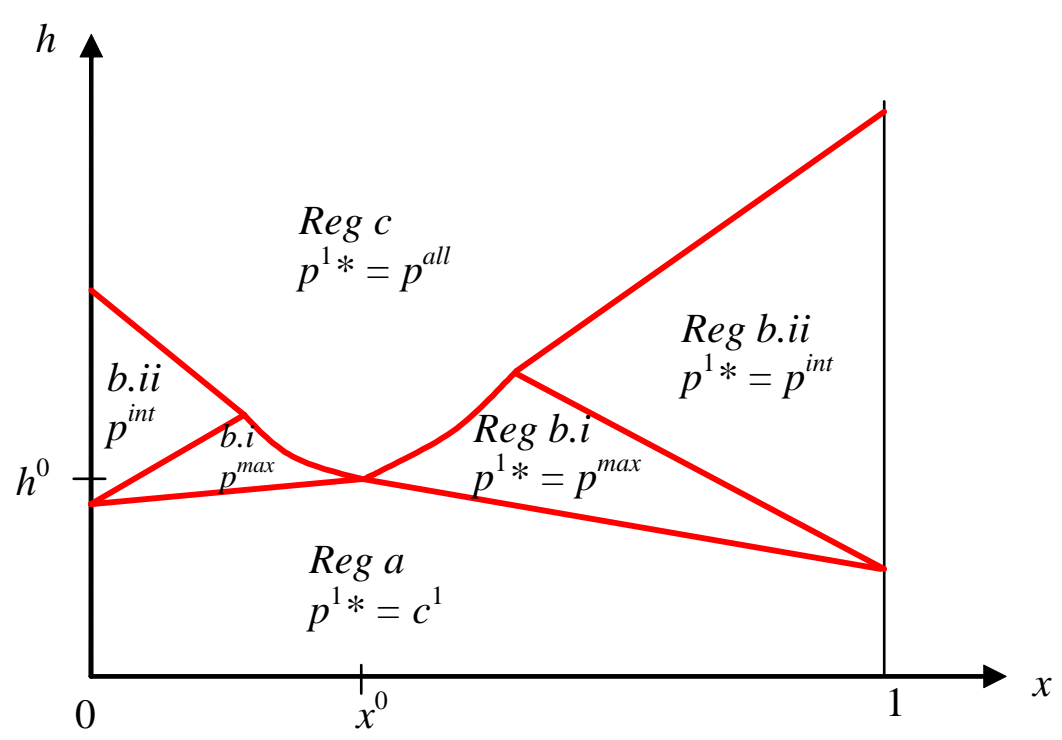

Figure 2: Optimal pricing policy by firm 1 in the drug space $(x, h)$ when $c^{1}=c^{0}$.

precisely, in Region a, where the "aggregate" difference $\Delta_{y}+l \Delta_{x}$ between the two drugs is not positive, firm 1 makes zero profit.

On the contrary, in Region c the cost-effectiveness of the new drug far outperforms that of the pioneer and firm 1 decides to set a price for which the new drug is prescribed to all patients. In this case, the price that allows the firm to serve the entire market $p^{\text {all }}$ is large enough so that for the firm it is worth setting $p^{\text {all }}$ instead of increasing the price further and losing some patients.

Finally, for intermediate values of $\Delta_{y}$ (Region b, which only exists if $\Delta_{x}>0$ ), the optimal price decision by firm 1 depends on the size of submarket $M$. If $M$ is sufficiently large, then firm 1 sets the maximum price compatible with selling the drug $p^{\max }$ because the number of patients that are treated with the new drug under this price is fairly large. In contrast, for low values of $M$, firm 1 would sell the drug to too few patients at $p^{\text {max }}$ and, therefore, it prefers to cut the price and it sets $p^{\text {int }}$.

Figure 2 represents the optimal pricing policy by the innovative firm for every combination of $\left(x^{1}, h^{1}\right)$, for a given value of $\Delta_{c}, b$ and $l$. The figure is drawn for $\Delta_{c}=0$. For a positive (resp. negative) $\Delta_{c}$, all the figure would move upwards (resp. downwards) in a proportion $\frac{1}{b} \Delta_{c}$. Figure 2 is not symmetric with respect to the vertical $x^{0}$ because, in this example, $x^{0} \neq \frac{1}{2}$.

It is worth noticing that although the optimal price decision $p^{1 *}$ of firm 1 is continuously increasing in the quality of the drug (i.e., in $\Delta_{y}$ ) inside each region, it is neither continuous nor increasing when $\Delta_{y}$ moves from Region b to Region c. At the border of the two regions, 
where $\Delta_{y}=\lg \left(\Delta_{x}\right), p^{\max }>p^{\text {all }}$ (in Region b.i) and $p^{\text {int }}>p^{\text {all }}$ (in Region b.ii). Therefore, the price $p^{1 *}$ decreases from either $p^{\max }$ or $p^{\text {int }}$ to $p^{\text {all }}$ if a marginal increase in $\Delta_{h}$ moves the drug from Region b to Region c. On the other hand, the price $p^{1 *}$ is continuous in $\Delta_{y}$ (and in $\Delta_{x}$ ) at the border of the Region b.i and Region b.ii. Finally, the optimal price $p^{1 *}$ increases with the horizontal differentiation $\Delta_{x}$ in Region b.i, but decreases with $\Delta_{x}$ in Region b.ii and Region c.

Corollary 1 provides some comparative statics of firm 1's profits with respect to the level of differentiation between the two drugs, $\Delta_{x}$ and $\Delta_{h}$. We will use these expressions in section 6 and subsection 7.3 where we compare the private and social incentives to develop a new drug.

Corollary 1 The comparative statics of firm 1's profits are as follows:

Region b.i: $\Pi^{1}$ is increasing and linear in $\Delta_{y}$. It is a concave function in $\Delta_{x}$, increasing up to $\Delta_{x}=\frac{1}{2 l}\left(l M-\Delta_{y}\right)$ and decreasing afterwards.

Region b.ii: $\Pi^{1}$ is increasing and convex in $\Delta_{y}$ and it is decreasing and convex in $\Delta_{x}$.

Region c: $\Pi^{1}$ is increasing and linear in $\Delta_{y}$ and it is decreasing and linear in $\Delta_{x}$.

Corollary 1 shows that the profits of the innovative firm are always larger the more cost-effective the new drug is -that is, the larger the difference $h^{1}-h^{0}$ and/or the smaller the difference $c^{1}-c^{0}$. Regarding the horizontal characteristic of the drug, firm 1's profits are decreasing in $\Delta_{x}$, except in Region b.i, where they are increasing for low values of $\Delta_{x} \cdot{ }^{14}$

\section{Benefit to the Health System}

The launch of the new drug affects the benefit of the health system. We now move to discuss the health system surplus in the different price regions.

Recall that in our model the doctor is a perfect agent for the health system. Therefore, he only prescribes the new drug when the surplus of the health system is higher than, or equal to, the surplus obtained using the pioneer drug. We denote by $\triangle C S$ the gain

\footnotetext{
${ }^{14} \mathrm{An}$ increase in the horizontal differentiation between the two drugs has two opposite effects on the profits of firm 1. On the one hand, for a given price, the market share of firm 1 decreases because more patients prefer the pioneering drug, which has a negative impact on the profits of the firm. On the other hand, a larger differentiation allows the firm to increase the price, which may have a positive impact on its profits. In relative terms, the positive effect of the price is less and less important as the demand for the new drug decreases, that is, as the horizontal differentiation increases. In our model, the positive effect only outweighs the negative one when $\Delta_{x}$ is small.
} 
in patient health, net of the price, as a consequence of the launch of the new drug. Proposition 2 provides the expressions for $\triangle C S$ in the regions identified in Proposition 1.

Proposition 2 Given the optimal pricing policy $p^{1 *}$ of firm 1, the variation of the health system surplus $\triangle C S$ due to the launch of the new drug is:

Region a: $\triangle C S\left(c^{1}\right)=0$.

Region b.i: $\Delta C S\left(p^{\max }\right)=0$.

Region b.ii: $\Delta C S\left(p^{i n t}\right)=\frac{b}{16 l}\left(\Delta_{y}+3 l \Delta_{x}-2 l M\right)\left(\Delta_{y}-5 l \Delta_{x}+6 l M\right)$.

Region c: $\Delta C S\left(p^{\text {all }}\right)=b l \Delta_{x}\left(2 M-\Delta_{x}\right)$.

Some interesting insights can be extracted from Proposition 2. In the scenario where the new drug is sold at $p^{\max }$ (Region b.i), total surplus is the same before and after the launch of the new drug. The reason is that the firm charges the largest potential price, $p^{\max }$, and it therefore extracts all the surplus from the patients that in equilibrium are prescribed the new drug. In contrast, in Region b.ii, the firm decides to charge $p^{\text {int }}<p^{\max }$ to attract some new patients and, thus, the health system surplus increases when the new drug is marketed. Finally, in the scenario where drug 1 takes over the market (Region c) the health system also benefits from the launch of the new drug when $\Delta_{x}>0$. In this case, the health advantages of the new drug outweighs the larger price charged by the innovative firm, so that the health system ends up strictly better off.

We also provide some comparative statics of the health system surplus with respect to the level of differentiation of the two drugs, $\Delta_{x}$ and $\Delta_{y}$.

Corollary 2 The comparative statics of the health system surplus are as follows:

Regions a and b.i: $\triangle C S$ does not change with $\Delta_{y}$ or $\Delta_{x}$.

Region b.ii: $\Delta C S$ is increasing in $\Delta_{y}$. It is increasing up to $\Delta_{x}=\frac{1}{15 l}\left(14 l M-\Delta_{y}\right)$ and then decreasing in $\Delta_{x}$.

Region c: $\triangle C S$ is independent of $\Delta_{y}$ and it is increasing in $\Delta_{x}$.

Moreover, $\triangle C S$ is continuous at the border between Region b.i and Region b.ii but it discontinuously increases when $\Delta_{y}$ increases (or $\Delta_{x}$ decreases) and the drug moves from Region b to Region $c$.

Corollary 2 shows that the gains derived by the health system from the launch of the new drug depend on the cost-effectiveness of this drug as compared to the pioneering drug (i.e., on $\Delta_{y}$ ) only in Region b.ii. In this region, the higher the cost-effectiveness 
(either due to advantages in quality or costs) of the new drug, the higher the gain in the surplus. In the other regions, a larger cost-effectiveness of the drug is fully translated into a higher price. Notice that health system surplus is certainly more sensitive to the horizontal characteristic $\Delta_{x}$. In particular, it tends to be increasing in $\Delta_{x}$, except in the region where the new drug replaces the pioneer for some patients (Region b.ii), where the health system surplus is decreasing for high values of $\Delta_{x}$. As $\Delta_{x}$ increases, the innovative firm cannot appropriate all the surplus through a higher price because its market would be too reduced.

\section{Private versus Social Incentives}

Before analyzing the innovation stage (in section 7), we briefly discuss here the difference in incentives for $R \& D$ between the innovative firm and a social planner who takes into account the sum of the health system surplus and the firm's benefits.

$\mathrm{R} \& \mathrm{D}$ incentives in our model come from the benefits that the parties involved derive from the launching of the potential new drug. To discuss the difference between private and social $R \& D$ incentives, it is worth noting that the innovative firm and the health system have different preferences regarding the characteristics of their best drug, given the pioneering drug on the market. Figure 3 represents the comparative statics of firm 1's profits and health system surplus with respect to vertical and horizontal changes in the new drug as compared to the pioneer (corollaries 1 and 2). ${ }^{15}$ In general, the firm cares a great deal about increasing the quality of the drug, either through a more effective or a less costly drug, whereas the health system (as it takes the price into account) only benefits from the launching of a better-quality drug if such a drug is well-suited to those patients far from $x^{0}$ (Region b.ii). Additionally, the profits of the innovative firm decrease with the degree of horizontal differentiation between the two drugs, except when the differentiation is small and the quality of the two drugs is similar (the left-hand part of Region b.i). On the contrary, the health system always benefits if new drugs focus on patients for whom the effectiveness of the old drug is low.

Figure 3 helps us to better understand the difference between incentives to innovate for the firm and for a social planner. For example, if the innovative firm could launch a drug through a deterministic process then it would have an incentive to choose a drug with a higher quality but the same horizontal characteristic as the pioneering drug. In fact, if the new drug were available at no cost (or at a low cost), the optimal drug for firm 1 would

\footnotetext{
${ }^{15}$ Figure 3 only represents the comparative statics for drugs on the right-hand side of $x^{0}$; for those drugs on the left-hand side of $x^{0}$ the comparative statics are similar. Moreover, the figure does not point out the effects that are zero. For example, in Region c, $\Delta C S$ is independent of $\Delta_{y}$ and the figure does not include information about the behavior of $\Delta C S$ with respect to $\Delta_{y}$ in that region.
} 


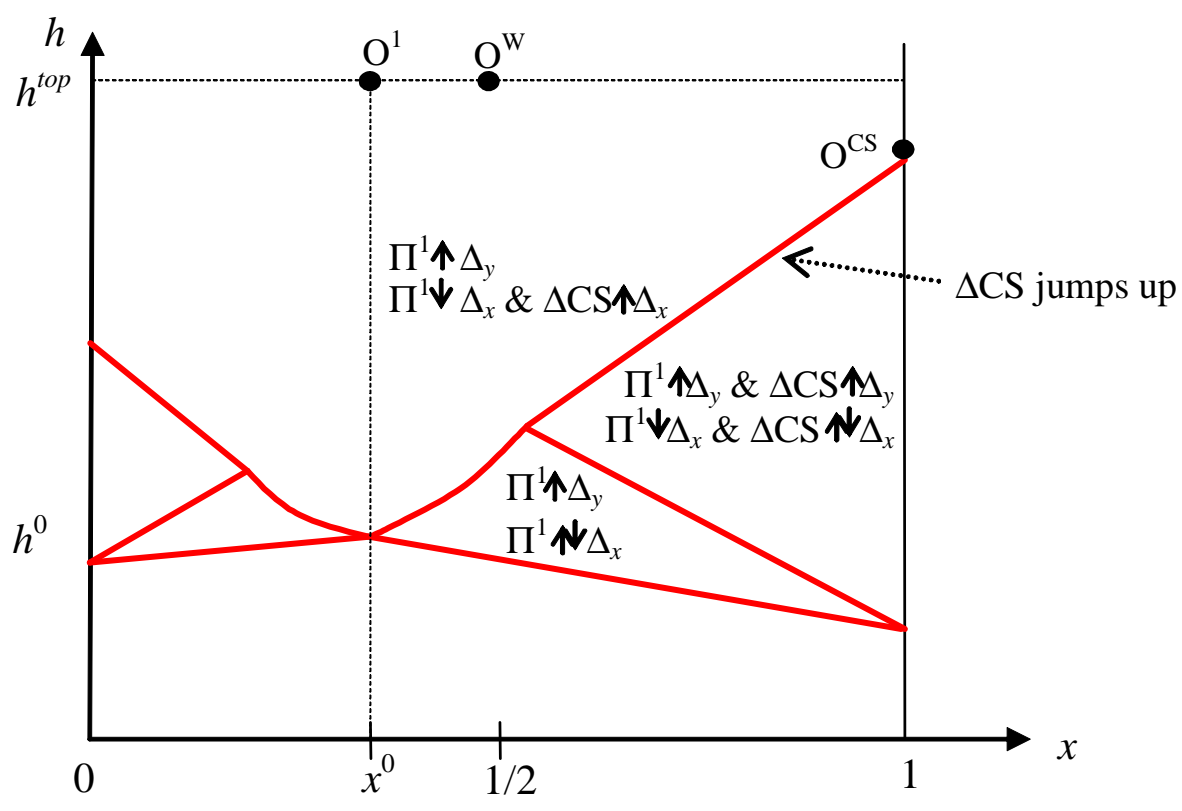

Figure 3: Comparative statics and most preferred drugs in the drug space $(x, h)$.

be $\mathrm{O}^{1}=\left(x^{0}, h^{\text {top }}\right)$ in Figure 3 , where $h^{\text {top }}$ is the highest possible quality. In contrast, consumers prefer drugs that are horizontally differentiated from the pioneering drug. In terms of consumer surplus, the optimal drug would be $\mathrm{O}^{C S}$, the farthest from $x^{0}$ in the horizontal dimension and at the border of Region c. Finally, if we define the increase in social welfare as the sum of firm 1's profits and the increase in the health system surplus, ${ }^{16}$ that is, $\Delta W \equiv \Pi^{1}+\Delta C S$, then balancing both firm and consumer interests, the optimal drug from a total welfare point of view would be $\mathrm{O}^{W}$ in Figure 3. The drug $\mathrm{O}^{W}$ improves upon the pioneering drug as much as possible in the vertical dimension and it is also horizontally differentiated from the pioneering drug, although the optimal differentiation is far from the maximal. In fact, the drug $\mathrm{O}^{W}$ would be horizontally located at $1 / 2$ since this is the drug type that minimizes the side-effects of the whole population of patients.

\section{$7 \quad$ A Model of Pharmaceutical Innovation}

In this section we analyze the first stage of our game, the innovation stage, where firm 1 chooses its R\&D investment to maximize its expected profit. The innovation strategy of the firm involves both the type of innovative process that it wants to adopt and the level of the resources invested in that process. We now describe a typology of drugs and

\footnotetext{
${ }^{16}$ We have assumed that the pioneering drug is sold at its marginal cost. Thus, the firm (or any of the firms) selling drug 0 always has zero profits.
} 
innovation processes and then discuss the firm's innovation decision.

\subsection{Me-Too versus Breakthrough Drugs}

In the pharmaceutical industry, a crucial distinction is made between me-too and breakthrough innovations. The traditional distinction between these two types of innovation relies on the mechanism of the action of the drugs. However, several voices in the pharmaceutical industry have called for the adoption of a broader perspective when evaluating innovation in medicines. ${ }^{17}$ Our interpretation is that the difference between me-too and breakthrough innovations rests on the distance between the new drug and the pioneer at either the horizontal axis and/or the vertical one. A me-too innovation represents a small change for some (or all) patients either in terms of quality of treatment, cost savings or side effects. For example, a me-too innovation may open the possibility of administering smaller or fewer doses, or it might imply a slightly less invasive delivery (which we interpret as a small increase in $y) .{ }^{18} \mathrm{~A}$ me-too innovation may also cause slightly lower side effects for a subpopulation of patients (a small change in $x$ ). ${ }^{19}$ In contrast, a breakthrough innovation ensures a significant increase in the quality of the new drug or a drug whose characteristics make it well-suited to patients who could not be well treated under the existing treatment. ${ }^{20}$

\subsection{Incremental versus Radical Innovation Processes}

A firm seeking a me-too or a breakthrough innovation would rely on different processes. In this sense, we define an incremental innovation process as a process aiming at a metoo innovation with respect to the existing drug. ${ }^{21}$ Similarly, when the process pursues a significant improvement (for all or a subpopulation of patients), or a substantial cost reduction as compared to the old drug, then we say that the innovation process is radical.

\footnotetext{
${ }^{17}$ See the report by Mestre-Ferrandiz, Mordoh, and Sussex (2012) for a thorough discussion of the nature of innovation in medicines.

${ }^{18}$ Insulin pens, for instance, are minimally invasive and have largely superseded the conventional insulin syringe.

${ }^{19}$ The antihistamine Clarinex (desloratidine), for instance, is a small variation of Claritin (loratidine) that has lower side effects for those people with severe allergies and/or accompanying chronic respiratory problems.

${ }^{20}$ For instance, Prilosep, based on the first proton pump inhibitor (omeprazole), was considered a breakthrough. It proved to be more effective than histamine-2 receptor antagonists to the management of peptic ulcer diseases.

${ }^{21}$ Sometimes, follow-on drugs are simply the natural outcome of simultaneous research programs into the same therapeutic target (diMasi and Paquette, 2004). In other cases, they are the result of an intentionally imitative research program (Garnier, 2008). The approach adopted in this paper is best suited to this second view of the innovation process.
} 
Formally, an incremental innovation process (which we will denote by the sub-index in) pursues a drug with a small $\Delta_{x}$ or $\Delta_{y}$ whereas a radical innovation process (denoted by the sub-index $r a$ ) aims at a drug with a large $\Delta_{x}$ and/or $\Delta_{y}$.

\subsection{A Simple Model of Innovation}

In this subsection we propose a highly stylized model of $R \& D$ investment with uncertainty. We assume that the result of the innovation process is uncertain and its outcome can only be poorly predicted, if at all. The uncertainty is greater for groundbreaking processes.

There are two types of innovation strategies available to the pharmaceutical firm. An incremental innovation process can lead to a drug that is similar to the pioneering drug, in the sense that it would have the same horizontal (or vertical) characteristic, that is, $x^{1}=x^{0}$ (or $y^{1}=y^{0}$ ) and a minor improvement in quality (or small differences in side effects). ${ }^{22}$ On the other hand, a radical innovation process results, in the case of success, in a drug $\left(x^{1}, y^{1}\right)$ where $y^{1}$ is random but higher than $y^{0}$ and $x^{1}$ is also random. This reflects the idea that the innovative process represents a significant departure from the old drug. Moreover, the side effects may also be very different from those of the pioneering drug. We depict the two categories of processes in Figure $4 .{ }^{23}$ The crucial differences between the two categories of processes is that the distribution of the outcome of a radical innovation process takes values on a large support and has a greater variance, which leads to higher chances of getting a breakthrough drug (Girotra, Terwiesch, and Ulrich, 2007; Singh and Fleming, 2010).

Let us first consider incremental innovation processes. If an investment $I$ is realized then there is a probability $q_{i n}(I)$, increasing in $I$, of obtaining an innovation, while no drug is obtained with probability $1-q_{i n}(I)$. If it is a vertical innovation process then, in the case of success, the new drug has characteristics $\left(x^{1}, y^{1}\right)$, with $x^{1}=x^{0}$ and $y_{1}$ is distributed according to the density function $f_{\text {in }}\left(y^{1}\right)$ that takes values in the interval $\left[y^{0}-\gamma_{v e r}, y^{0}+\delta_{v e r}\right]$, where $\gamma_{v e r}$ and $\delta_{v e r}$ are small (in the sense that the new drug cannot be much more cost-effective than the pioneer). Thus, for all $I$ invested in a vertical

\footnotetext{
${ }^{22}$ Sometimes the incremental innovation process is developed by the same firm that holds the patent for the pioneering drug in order to better compete with those firms offering generic versions of the drug once the patent has expired. Examples are Prilosec and Nexium, aimed at decreasing the amount of acid produced in the stomach. In 2001 the patent for Prilosec (which has omeprazole as an active ingredient), owned by AstraZeneca, expired and several generic versions of omeprazole entered the market. The same year, Nexium, whose active ingredient is esomeprazole (a version of omeprazole whose molecular orientation is left-handed), was also patented by AstraZeneca (our firm 1).

${ }^{23}$ Note that our innovation process in the vertical dimension differs notably from the vertical innovation analyzed by Bardey et al. (2010), where a vertical innovation is always synonymous with a breakthrough innovation.
} 


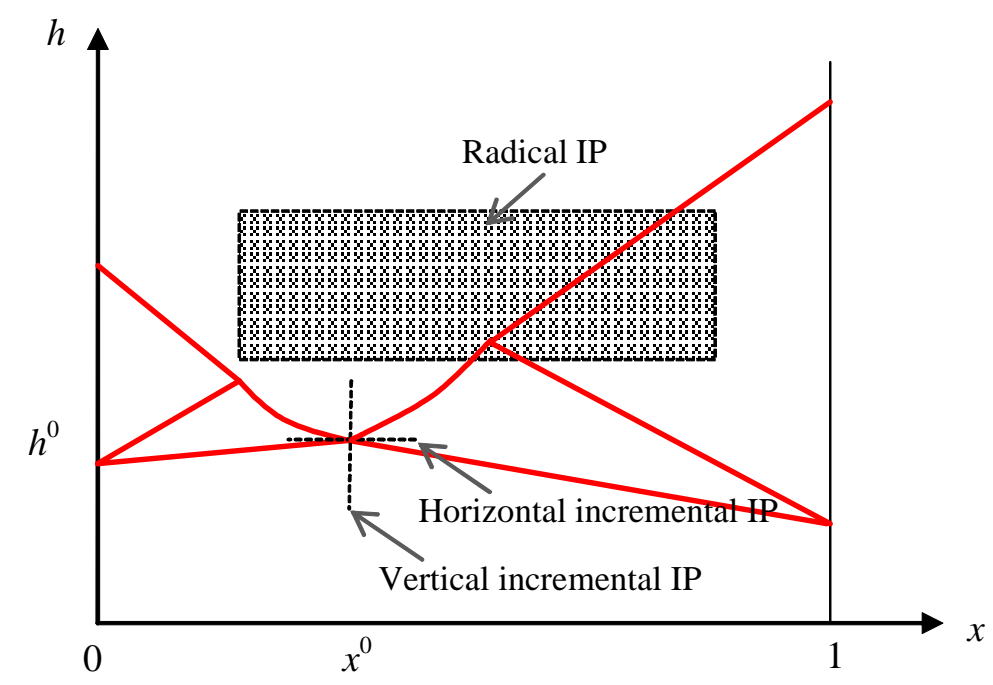

Figure 4: Incremental and radical innovation processes.

incremental innovation process, expected private profits $\Pi_{i n}(I)$ and social welfare $W_{i n}(I)$ are

$$
\begin{gathered}
\Pi_{i n}(I)=q_{i n}(I) \int_{\left[y^{0}-\gamma_{v e r}, y^{0}+\delta_{v e r}\right]} \Pi^{1}\left(x=x^{0}, y\right) f_{i n}(y) d y \\
W_{i n}(I)=q_{i n}(I) \int_{\left[y^{0}-\gamma_{v e r}, y^{0}+\delta_{v e r}\right]}\left(\Pi^{1}\left(x=x^{0}, y\right)+\Delta C S\left(x=x^{0}, y\right)\right) f_{i n}(y) d y,
\end{gathered}
$$

where $\Pi^{1}(x, y)$ and $\Delta C S(x, y)$ denote the firm 1's profits and the increase in consumer surplus when the new drug has characteristics $(x, y)$.

The expressions for profits and social welfare are similar for horizontal incremental processes. In this case, the $\mathrm{R} \& \mathrm{D}$ investment leads, in the case of success, to a drug $\left(x^{1}, y^{1}\right)$ with $y^{1}=y^{0}$ and $x^{1}$ is distributed according to a density function $h_{i n}(x)$ that takes values in the interval $\left[x^{0}-\gamma_{h o r}, x^{0}+\delta_{h o r}\right]$, where $\gamma_{h o r}$ and $\delta_{h o r}$ are positive and small (in the sense that the new drug would be suitable for similar types of patient than the pioneer drug).

Proposition 3 compares private and social incentives to adopt an incremental innovation process.

Proposition 3 For an incremental innovation process pursuing a me-too drug, the optimal investment decision for firm $1, I_{\text {in }}^{1}$, and for the social planner, $I_{i n}^{*}$, coincide: $I_{\text {in }}^{1}=I_{i n}^{*}$.

The intuition for this result derives from the fact that, for drugs close to the pioneering drug, the innovative firm is always able to extract all the health system surplus. Consider a vertical innovation process. If it is successful and the drug has a characteristic $y^{1}>y^{0}$, 
firm 1 gets some monopoly power because the new drug lies in Region c. The firm will impact all the extra quality of the drug into a higher price and it is able to extract all the health system surplus $\left(\Delta C S=0\right.$ in Region c when $\left.\Delta_{x}=0\right)$. As the health system never benefits from the new drug, social and private incentives to innovate coincide as do their optimal investment levels. The intuition for the horizontal incremental process is similar: when the realization of $\tilde{x}^{1}$ is close to $x^{0}$, firm 1 sets the maximum price that the health authority is willing to pay and it extracts all the surplus $(\triangle C S=0)$.

We now consider a radical process. If an investment $I$ is made then there is a probability $q_{r a}(I)$, increasing in $I$, of success at getting a new drug $\left(x^{1}, y^{1}\right) .{ }^{24}$ If the process is successful, the new drug has characteristics $\left(x^{1}, y^{1}\right)$, where $y^{1}$ takes values in the interval $\left[y^{0}+\kappa, y^{0}+v\right]$ and $x^{1}$ takes values in the interval $[0,1]$, where $\kappa$ can be positive or negative, $v$ is positive, $\kappa<v$ and $\left(x^{1}, y^{1}\right)$ is distributed according to a density function $f_{r a}\left(x^{1}, y^{1}\right) .{ }^{25}$ Therefore, expected private and social benefits for each level of investment $I$ in a radical innovation process are

$$
\Pi_{r a}(I)=q_{r a}(I) \int_{[0,1]} \int_{\left[y^{0}+\kappa, y^{0}+v\right]} \Pi^{1}\left(x^{1}, y^{1}\right) f_{r a}\left(x^{1}, y^{1}\right) d y^{1} d x^{1}
$$

and

$$
W_{r a}(I)=q_{r a}(I) \int_{[0,1]} \int_{\left[y^{0}+\kappa, y^{0}+v\right]}\left(\Pi^{1}\left(x^{1}, y^{1}\right)+\Delta C S\left(x^{1}, y^{1}\right)\right) f_{r a}\left(x^{1}, y^{1}\right) d y^{1} d x^{1} .
$$

Proposition 4 states the difference between private and social incentives to invest in a radical innovation process.

Proposition 4 For a radical innovation process pursuing a breakthrough drug the optimal investment decision for firm $1, I_{r a}^{1}$, is smaller than that for the social planner, $I_{r a}^{*}: I_{r a}^{1}<$ $I_{r a}^{*}$.

A radical innovation process leads, with a certain probability, to a drug that improves the health system surplus. First, the health system benefits when the process is successful if the new drug lies in Region $\mathrm{c}$ and $x^{1} \neq x^{0}$. In this case, the innovative firm cannot appropriate all the surplus: at the maximum price at which it can serve all the market $\left(p^{\text {all }}\right)$, some patients are strictly better-off with the new drug than with the pioneer. Similarly, if the successful process leads to a drug in Region b.ii then the interior price $p^{\text {int }}$ allows the health system to benefit. A social planner would also take into account

\footnotetext{
${ }^{24}$ Typically, $q_{r a}(I)$ would be (much) lower than $q_{i n}(I)$ for any $I$.

${ }^{25}$ The function $f_{r a}\left(x^{1}, y^{1}\right)$ may not take values for some intervals of characteristics. Figure 4 , for instance, illustrates a process that never leads to very extreme drugs on the horizontal axis. In this figure, $\kappa>0$ but this is not necessary in general.
} 
this surplus that the firm cannot extract from the health system and it would choose a higher investment level than the firm.

As shown in propositions 3 and 4, the firm fully appropriates all the benefits derived from me-too drugs, whereas the health system can appropriate some surplus if a radical innovation process goes successfully. Proposition 5 uses these results to state the main policy implication of our analysis of the innovation incentives.

Proposition 5 If firm 1 can only adopt one category of innovation process (due, for instance, to financial or capacity constraints) then:

(i) There are cases where firm 1 prefers to adopt an incremental innovation process whereas the social welfare is larger when adopting a radical innovation process.

(ii) There is no case where firm 1 prefers to adopt a radical innovation process whereas adopting an incremental innovation process would generate a higher welfare.

Proposition 5 provides some theoretical support to the social concern that pharmaceutical firms devote too many resources to market me-too drugs and too few to launch breakthrough drugs. Our model suggests that this disalignment between private and social incentives is due to the lack of private incentives to pursue radical innovations. While private and social incentives to devote resources to pursue me-too drugs are aligned because the firm is able to appropriate all the benefits through the price, this is not true for first-in-class drugs. In the latter case, the firm sets a price for the new drug which is low enough to serve all (or a good part of) the market and the consumers benefit from it.

\section{The Physician is a Perfect Agent for the Patients}

In the previous sections we have assumed that the demand for the drugs is decided by a physician who prescribes the drug that provides the highest benefit to the whole health system. In this section we discuss the robustness of the results when the physician acts as a perfect agent for the patients, prescribing the drug that maximizes the patients' utility. Thus, the physician does not consider the whole cost of the drugs; he only internalizes the cost disbursed by the patient. ${ }^{26}$ We analyze two different reimbursement regimes: one in which patients pay a proportion of the price of the drugs not covered by health insurance (that is, there is a copayment), and a second one in which the old drug is fully financed by the health authority while the new one is not covered by health insurance.

\footnotetext{
${ }^{26}$ If the physician makes choices considering patient utility and health authority costs then we would be in the framework analyzed in the previous sections: the physician internalizes the whole price, regardless of whether they are paid by the health authority or by the patients themselves.
} 


\subsection{Patient Cost-Sharing of the Drugs}

Suppose that there is a copayment $\alpha \in(0,1]$ so that patients are required to pay $\alpha p$ if the price of the drug is $p$. Then, the benefit to the health system of treating patient $x$ with drug $(\hat{x}, \hat{h})$ and paying price $\hat{p}$ for this drug is $H(x ; \hat{x}, \hat{h}, \hat{p})$ while patients' utility, which determines the physician's prescription of drugs, is

$$
U(x ; \hat{x}, \hat{h}, \hat{p}) \equiv b(\hat{h}-l|\hat{x}-x|)-\alpha \hat{p}=\alpha\left[\frac{b}{\alpha}(\hat{h}-l|\hat{x}-x|)-\hat{p}\right] .
$$

If the physician follows the function $U(x ; \hat{x}, \hat{h}, \hat{p})$ instead of $H(x ; \hat{x}, \hat{h}, \hat{p})$ to make his choices, then all the results concerning physician prescription choices and firm 1's pricing decisions for the case $\alpha=1$ (Proposition 1) would remain valid for any $\alpha$ by replacing, in the expressions of $p^{\max }, p^{\text {all }}$, and $p^{\text {int }}$, the parameter $b$ by $\frac{b}{\alpha}$. That is, the results in a model where patients pay a proportion of the prices are similar to those in a model without copayment where the marginal utility of being healthy is higher. Since $\operatorname{sign}\left(\frac{\partial p^{1}}{\partial \alpha}\right)=$ $-\operatorname{sign}\left(\frac{\partial p^{1}}{\partial b}\right)$, and $p^{\max }, p^{\text {all }}$, and $p^{\text {int }}$ are all increasing in $b$, the price of the new drug will be higher the lower the copayment $\alpha .^{27}$

In order to examine the results more deeply we need to distinguish two cases. In the case $c_{1}=c_{0}$ (i.e., $\Delta_{c}=0$ ), the regions in the drug space depicted in Figure 2 are independent of the parameter $b$. Therefore, at the optimal prices, the new and the old drugs are prescribed to the same patients for any value of $\alpha$, although the price for drug 1 is larger as $\alpha$ decreases. This implies that, for $\Delta_{c}=0$, an increase in the copayment (that is, a decrease in $\alpha$ ) leads to a lower benefit to the health system and to a higher profit to firm 1 . However, such increase in the copayment has no impact on total welfare because changes in $\alpha$ only induce changes in the monetary transfers between the health system and the innovative firm. Thus, the optimal drug for firm 1 would still be $O^{1}$ (see Figure 3), the drug $O^{W}$ would still be optimal from a total welfare point of view (defined as the sum of the benefits of the health system and firm 1's profits), and the point $O^{C S}$ would correspond to the optimal drug for the patient (that is, the drug that maximizes the function $U)$.

In the case $c_{1}>c_{0}\left(\Delta_{c}>0\right)$, the borders of the regions in Figure 2 shift vertically down, and the new drug is prescribed to more patients with than without copayment. Here, the increase in the monetary transfer from the health authority to firm 1 due to the copayment comes combined with a misallocation of drugs from the health system

\footnotetext{
${ }^{27}$ This extension also corresponds to the case where there is no copayment but the physician gives different weights to the patient health (a weight of 1) and to the drugs' price (a weight of $a$ ). Equivalently, the model corresponds to a situation where the physician takes into account both the patients' utility and the profits of the health authority and puts less weight on the second term.
} 
perspective. The location of the optimal drugs $O^{1}, O^{W}$, and $O^{C S}$ in the drug space would be the same as in the case $\Delta_{c}=0$ when Figure 3 is drawn using the new (shifted-down) borders.

Finally, given that equilibrium prices are higher when the physician is a perfect agent for patients subject to copayment than when he is a perfect agent for the health system, the profits of the innovative firm are higher: the three functions $\Pi^{1}\left(p^{\max }\right), \Pi^{1}\left(p^{i n t}\right)$, and $\Pi^{1}\left(p^{\text {all }}\right)$ are linear in $b$; hence, they increase as $\alpha$ decreases. Therefore, firm 1's incentives to innovate are also higher. This result holds both for incremental and radical innovation processes. When $\Delta_{c}=0$, the regions in Figure 2 do not vary with $\alpha$ and, given the linearity of the profit functions in $b$, the trade-off between the two types of processes is unaffected: the firm has relatively too many incentives to pursue an incremental rather than a radical innovation process. If $\Delta_{c}>0$, low values of $\alpha$ imply that drugs close to the pioneer (in terms of $h$ and $x$ ) that would not have been marketed without copayments are now prescribed. Therefore, firm 1 has a stronger incentive to develop drugs whose additional therapeutic value is low, which suggests that it is yet more inclined to devote resources to incremental rather than to radical innovation processes.

\subsection{The New Drug is Not Subsidized}

Suppose now that the health authority only covers the old drug, so that patients have to pay the full price of the new one, should it be prescribed. Thus, patients' utility when treated with the old drug is

$$
U\left(x ; x^{0}, h^{0}, 0\right)=b\left(h^{0}-l\left|x^{0}-x\right|\right),
$$

while it takes the form

$$
U\left(x ; x^{1}, h^{1}, p^{1}\right)=b\left(h^{1}-l\left|x^{1}-x\right|\right)-p^{1}
$$

when patients are prescribed the new drug. Then, physician prescription choices and pricing decisions by the firm are obtained by considering the analysis conducted in the previous sections for the particular case $c_{0}=0$. Both the physician and the firm behave as if the price of the pioneering drug were 0 . Since $p^{\max }, p^{\text {all }}$, and $p^{\text {int }}$ are all decreasing in $c_{0}$, firm 1 is induced to choose lower prices than in our initial model. Moreover, looking at Figure 2, reductions in $c_{0}$ make the borders of the regions shift vertically up and, at the optimal prices, the new drug is prescribed to fewer patients as compared to the situation in which the cost of the pioneering drug is also taken into account. Thus, in this case, the firm obtains lower profits and it has lower incentives to innovate. However, its relative incentives to adopt radical innovation processes are now larger because me-too drugs do not provide any profits. 


\section{Conclusion}

The proliferation of follow-on drugs is nowadays the subject of some debate. Proponents of follow-on drugs highlight that some of them are therapeutically superior to the pioneer. Moreover, patients and physicians benefit from the access to a larger pool of therapeutic choices. But there are also voices warning that imitative drug development poses a threat, as it could reduce the incentive for firms to develop first-in-class drugs.

This paper seeks to contribute to this social debate. We have constructed a simple model where drugs are vertically and horizontally differentiated. After studying the optimal price decision of a firm introducing a new drug, we have analyzed the firm incentives to invest in $R \& D$ when a pioneer drug is already on the market. In particular, we have disentangled the quest for breakthrough drugs from the search of follow-on drugs. In our model, both breakthrough and follow-on drugs are socially valuable.

While private and social incentives to invest in $R \& D$ processes coincide for incremental innovation ventures, private incentives are lower than social ones when the process is radical. Moreover, we interestingly find that pharmaceutical firms are too prone to devoting resources (if scarce) to pursue incremental innovation processes so as to fully appropriate all the benefits derived from me-too drugs. Thus, these results somehow reproduce the social concern that the main problem regarding the rapid increase of me-too drugs is that they diminish the incentives for innovation in pioneering drugs.

Our conclusions are obtained under some simplifying assumptions; we now discuss some of them. First, we have assumed that the pioneering drug is sold on the market at its marginal price. This hypothesis fits well in markets where the patent for the pioneering drug has already expired and several generic drugs have been introduced. However, there are other markets where the pioneering drug is sold by one firm at a price over its marginal cost. In such cases, the incumbent firm may react to the launch of a new drug by reducing the price of its drug. Price competition between the two firms will be fiercer the lower the horizontal distance between the two drugs. This seems to suggest that under price competition the incentives of the innovative firm to differentiate itself in the horizontal axis increase, which may translate into more incentives to adopt radical innovation processes. In addition to this, if the patent covering the pioneering drug has not expired yet, an incremental innovation would be more likely to infringe on the patent than a radical one, which would further increase the firm's incentives to invest in radical innovations.

Secondly, our innovative firm freely chooses the price of the new drug. However, regulations worldwide to control excessive market power of pharmaceutical firms abound. It would be worth investigating whether price regulations (such as price ceilings or reference prices) would be an effective tool to align the incentives of the firm and the society. Price

ceilings in our model, for instance, would undermine private incentives to innovate. Given 
that the difference in prices between the new and the pioneering drugs is higher for radical than for incremental innovations, the price ceilings should have a larger negative impact on radical than on incremental processes. Our analysis also suggests that price ceilings would have a larger impact on vertical rather than on horizontal innovations. Since vertical improvements allow the pharmaceutical firm to charge higher prices, the firm will be more constrained by the regulated price if it launches a new drug that is an improvement over the pioneer in the vertical dimension. Regarding reference prices, the restriction on prices might concern only those drugs that are close to the pioneer. In that case, the incentives for radical innovations may be less affected by the regulation than those for incremental innovations as more differentiated new drugs manage to charge a larger price and obtain higher profits.

Third, our results have been obtained under the assumption of risk neutrality for all the players involved. However, it is natural to think that pharmaceutical firms exhibit a certain degree of risk aversion. If we relax the risk neutrality assumption to accommodate more realism, our main result would be reinforced as risk aversion would make firms even more prone to adopt (safer) incremental innovations.

Finally, in our model there is only one innovative firm so we have not considered competition in research (in Bardey et al., 2010, several laboratories compete in the research sector of the pharmaceutical industry). If firms compete in adopting incremental innovation processes, they will overinvest in comparison to the socially efficient level, leading to more me-too drugs than the socially optimal number. At the same time, the innovative firm will also increase its investment in radical innovation processes under the pressure of competition. And this could lead to investment levels closer to the socially optimal one. Thus, if firms compete in research, the result that they devote too many resources to conducting R\&D activities that lead to incremental innovations still holds.

In this paper, we have identified a problem of misalignment between private and social incentives to innovate that results in a bias toward me-too drugs. A more normative analysis, in which different solutions to the problem can be addressed, is left for further research. Such policies could include direct R\&D tax incentives, nonprofit tax exemptions for research institutions, public financing of R\&D activity, as well as many other instruments that attempt to stimulate various forms of research and innovative activity. Our analysis suggests that the optimal R\&D policy should focus on providing incentives for radical innovation ventures.

Finally, although there seems to be a great social awareness of the proliferation of me-too drugs, to date, economists have done little theoretical research on this issue. We hope that this study opens the door to further research into that area and that it will also stimulate the ongoing debate over the excessive launching of me-too drugs. 


\section{References}

[1] Angell, M., 2004. The truth about the drug companies. New York: Random House.

[2] Antoñanzas, F., Juárez-Castelló, C., Rodríguez-Ibeas, R., 2011. Innovation, loyalty and generic competition in pharmaceutical markets. SERIEs 2, 75-95.

[3] Avorn, J., 2004. Powerful medicines. New York, Knopf.

[4] Bardey, D., Bommier, A., Jullien, B., 2010. Retail Price Regulation and Innovation: Reference Pricing in the Pharmaceutical Industry. Journal of Health Economics 29 (2), 303-316.

[5] Berndt, E. R., Aitken, M., 2011. Brand Loyalty, Generic Entry and Price Competition in Pharmaceuticals in the Quarter Century after the 1984 Waxman-Hatch Legislation. International Journal of the Economics of Business 18 (2), 177-201.

[6] diMasi, J., Paquette, C., 2004. The Economics of Follow-on Drug Research and Development Trends in Entry Rates and the Timing of Development. Pharmacoeconomics 22 (Suppl. 2), 1-14.

[7] European Commission, 2013. The 2012 EU Industrial R\&D Investment Scoreboard. European Commission. Joint Research Centre (JRC). Luxembourg: Publications Office of the European Union.

[8] García, R., Calantone, R., 2002. A Critical look at technological innovation typology and innovativeness terminology. A literature review. Journal of Product Innovation Management, 19(2), 110-132.

[9] Ganuza J., Llobet G., Domínguez B., 2009. R\&D in the Pharmaceutical Industry: A World of Small Innovations. Management Science 55 (4), 539-551.

[10] Garnier, J.P., 2008. Rebuilding the R\&D engine in big pharma. Harvard Business Review 86(5), 68-70, 72-76, 128.

[11] Girotra, K., Terwiesch, C., Ulrich, K.T., 2007. Valuing R\&D projects in a portfolio: Evidence from the pharmaceutical industry, Management Science 5 (9), 1452-1466.

[12] Goozner, M., 2004. The $\$ 800 \mathrm{~m}$ pill: The truth behind the cost of new drugs. Berkeley, University of California Press.

[13] Hernandez A.F., Harrington, R.A., 2008. Comparative effectiveness of angiotensinconverting-enzyme inhibitors: is an ACE always an ace? Canadian Medical Association Journal 178 (10), 1316-1319. 
[14] Kapur, N.K., Musunuru, K., 2008. Clinical efficacy and safety of statins in managing cardiovascular risk. Journal of Vascular Health and Risk Management 4(2), 341-353.

[15] Mestre-Ferrandiz, J., Mordoh, A., Sussex, J., 2012. The many faces of innovation. Office for Health Economics. Available at: http://www.ohe.org/publications/article/the-many-faces-of-innovation-119.cfm

[16] Miller, H.I., 2014. Critics of 'Me-Too Drugs' Need to Take a Chill Pill. Wall Street Journal, January 1st.

[17] Pharmaceutical Research and Manufacturers of America, 2013. 2013 Biopharmaceutical Research Industry Profile. Washington, DC, PhRMA, July.

[18] Singh, J., Fleming L., 2010. Lone Inventors as Sources of Breakthroughs: Myth or Reality? Management Science 56 (1), 41-56.

[19] Wertheimer, A.I., Santella T.M., 2009. Pharmaceutical Evolution: The Advantages of Incremental Innovation in Drug Development. Competitive Enterprise Institute. Issue Analysis, no.2, April. Washington, DC.

[20] Yin, N., 2012. Pharmaceuticals, Incremental Innovation and Market Exclusivity. Mimeo, Toulouse School of Economics.

\section{Appendix}

Proof of Proposition 1. We distinguish three zones of parameters: $c^{1} \geq p^{\max }$, $c^{1} \in\left(p^{\text {all }}, p^{\max }\right)$, and $c^{1} \leq p^{\text {all }}$. We note that $c^{1} \geq p^{\max }$ if and only if $\Delta_{c} \geq b\left(l \Delta_{x}+\Delta_{h}\right)$, i.e., $\Delta_{y} \leq-l \Delta_{x}$. Similarly, $c^{1} \leq p^{\text {all }}$ if and only if $\Delta_{c} \leq b\left(\Delta_{h}-l \Delta_{x}\right)$, i.e., $\Delta_{y} \geq l \Delta_{x}$. We analyze the optimal firm's pricing in the three zones.

Zone $\boldsymbol{\Delta}_{y} \leq-\mathbf{l} \boldsymbol{\Delta}_{x}$. Given that $c^{1} \geq p^{\max }$, there is no price above marginal cost at which the physician prescribes the new drug to some patients. Therefore, the best strategy for firm 1 is to not sell its drug.

Zone $\boldsymbol{\Delta}_{y} \in\left(-\mathbf{l} \boldsymbol{\Delta}_{x}, \mathbf{l} \boldsymbol{\Delta}_{x}\right)$. In this zone, $c^{1}>p^{\text {all }}$ and firm 1 cannot select a price at which it makes a profit by selling to all the patients. If $x^{1}>x^{0}$, the firm chooses the price $p^{1}$ that maximizes its profits, taking into account that its drug will be prescribed to those patients with characteristic $x \in[\tilde{x}, 1)$. Hence, $p^{1} \in\left[c^{1}, p^{\max }\right]$ maximizes

$$
\Pi^{1}\left(p^{1}\right)=\left(p^{1}-c^{1}\right)(1-\tilde{x})=\left(p^{1}-c^{1}\right)\left[1-x^{0}-\frac{\left(l \Delta_{x}-\Delta_{h}\right)}{2 l}-\frac{\left(p^{1}-c^{0}\right)}{2 b l}\right] .
$$


We write the expression for the profits as

$$
\Pi^{1}\left(p^{1}\right)=\left(p^{1}-c^{1}\right)\left[M-\frac{\left(l \Delta_{x}-\Delta_{h}\right)}{2 l}-\frac{\left(p^{1}-c^{0}\right)}{2 b l}\right],
$$

which is also valid for the situation where $x^{1}<x^{0}$ (if $x^{1}=x^{0}$, this zone is empty). ${ }^{28}$

The first-order and second-order conditions are

$$
\begin{gathered}
\Pi_{p^{1}}^{1}\left(p^{1}\right)=M-\frac{\left(l \Delta_{x}-\Delta_{h}\right)}{2 l}-\frac{\left(2 p^{1}-\left(c^{0}+c^{1}\right)\right)}{2 b l}, \\
\Pi_{p^{1} p^{1}}^{1}\left(p^{1}\right)=\frac{-1}{b l}<0 .
\end{gathered}
$$

The interior candidate to solution $p^{1}$ satisfies $\Pi_{p^{1}}^{1}\left(p^{1}\right)=0$ and is given by

$$
p_{1}=p^{i n t}=\frac{1}{2}\left(c^{0}+c^{1}\right)+b l M-\frac{1}{2} b\left(l \Delta_{x}-\Delta_{h}\right) .
$$

The concavity of the function $\Pi^{1}\left(p^{1}, \Delta_{x}, \Delta_{h}\right)$ implies that the candidate $p^{\text {int }}$ is the optimum if and only if it lies in the interval $\left[c^{1}, p^{\max }\right]$. First, $p^{\text {int }}>c^{1}$ if and only if

$$
\frac{1}{2} \Delta_{c}+b l M-\frac{1}{2} b\left(l \Delta_{x}-\Delta_{h}\right)>0,
$$

that is, $\Delta_{y}>l \Delta_{x}-2 l M$. Given that $M \geq \Delta_{x}$, the previous inequality is implied by $\Delta_{y}>-l \Delta_{x}$, which is satisfied in this zone. Second, $p^{i n t}<p^{\max }=c^{0}+b\left(l \Delta_{x}+\Delta_{h}\right)$ if and only if

$$
\frac{1}{2} \Delta_{c}+b l M-\frac{1}{2} b\left(l \Delta_{x}-\Delta_{h}\right)<b\left(l \Delta_{x}+\Delta_{h}\right),
$$

from which it easily follows that $p^{\text {int }}<p^{\max }$ if and only if $M<\frac{1}{2 l}\left(3 l \Delta_{x}+\Delta_{y}\right)$. Therefore, in this zone, $p^{1 *}=p^{i n t}$ if $M<\frac{1}{2 l}\left(3 l \Delta_{x}+\Delta_{y}\right)$ and $p^{1 *}=p^{\max }$ otherwise $\left(p_{1}=c\right.$ cannot be optimal because profits would be zero).

Zone $\boldsymbol{\Delta}_{y} \geq \mathbf{l} \boldsymbol{\Delta}_{x}$. In this zone, firm 1 can set the price $p^{\text {all }}$ that would allow it to attract all patients in $[0,1]$. It can also choose any price in the interval $p^{1} \in\left(p^{\text {all }}, p^{\text {max }}\right]$, in which case the new drug will be prescribed to a subset of the patients. In this interval, the interior candidate to solution is $p^{i n t}$, as in the previous zone. We check the conditions under which $p^{\text {int }} \in\left(p^{\text {all }}, p^{\max }\right]$.

First, $p^{\text {int }}>p^{\text {all }}$ if and only if $\frac{1}{2}\left(c^{0}+c^{1}\right)+b l M-\frac{1}{2} b\left(l \Delta_{x}-\Delta_{h}\right)>c^{0}+b\left(\Delta_{h}-l \Delta_{x}\right)$, that is,

$$
M>\frac{1}{2 l}\left(\Delta_{y}-l \Delta_{x}\right)
$$

\footnotetext{
${ }^{28}$ If $x^{1}<x^{0}$, then

$$
\tilde{x}=x^{0}-\frac{\left(l \Delta_{x}-\Delta_{h}\right)}{2 l}-\frac{\left(p^{1}-c^{0}\right)}{2 b l}=M-\frac{\left(l \Delta_{x}-\Delta_{h}\right)}{2 l}-\frac{\left(p^{1}-c^{0}\right)}{2 b l} .
$$
}


Note that at $p^{1}=p^{\text {int }}$, firm 1 serves a market of size $M$. However, this cannot be the best pricing strategy for the firm because it can serve the whole market and obtain larger profits by marginally decreasing the price. Therefore, if $M \leq \frac{1}{2 l}\left(\Delta_{y}-l \Delta_{x}\right)$, setting the price $p^{\text {all }}$ and serving the whole market is certainly the optimal decision.

Second, as we show in the analysis of Zone $\Delta_{y} \in\left(-l \Delta_{x}, l \Delta_{x}\right), p^{i n t} \leq p^{\max }$ if and only if $M \leq \frac{1}{2 l}\left(3 l \Delta_{x}+\Delta_{y}\right)$.

Thus, for $M \geq \frac{1}{2 l}\left(3 l \Delta_{x}+\Delta_{y}\right)$, the candidates for solution are $p^{\max }$ and $p^{\text {all }}$; for $M \in\left(\frac{1}{2 l}\left(\Delta_{y}-l \Delta_{x}\right), \frac{1}{2 l}\left(3 l \Delta_{x}+\Delta_{y}\right)\right)$ the candidates for solution are $p^{\text {int }}$ and $p^{\text {all }}$; and for $M \leq \frac{1}{2 l}\left(\Delta_{y}-l \Delta_{x}\right)$, the optimal price is $p^{\text {all }}$.

We now analyze the conditions for $\Pi^{1}\left(p^{\max }\right) \geq \Pi^{1}\left(p^{\text {all }}\right)$, that is, $b\left(M-\Delta_{x}\right)\left(\Delta_{y}+l \Delta_{x}\right) \geq$ $b\left(\Delta_{y}-l \Delta_{x}\right)$, or

$$
\Delta_{y} \leq \frac{\left(1+M-\Delta_{x}\right)}{\left(1-M+\Delta_{x}\right)} l \Delta_{x}=\lg \left(\Delta_{x}\right) .
$$

We note that the border $\Delta_{y}=l g\left(\Delta_{x}\right)$ always lies in the zone $\Delta_{y} \geq l \Delta_{x}$, because $b \Delta_{y}-$ $b l g\left(\Delta_{x}\right) \leq b\left(\Delta_{y}-l \Delta_{x}\right)$ if and only if $g\left(\Delta_{x}\right) \geq \Delta_{x}$, which is equivalent to $\frac{\left(1+M-\Delta_{x}\right)}{\left(1-M+\Delta_{x}\right)} \geq 1$, or $M \geq \Delta_{x}$, which holds.

Similarly, $\Pi^{1}\left(p^{\text {int }}\right) \geq \Pi^{1}\left(p^{\text {all }}\right)$ if and only if $\frac{b}{2 l}\left(l M+\frac{1}{2}\left(\Delta_{y}-l \Delta_{x}\right)\right)^{2} \geq b\left(\Delta_{y}-l \Delta_{x}\right)$ that, denoting $t \equiv\left(\Delta_{y}-l \Delta_{x}\right)$, can be written as $\left(l M+\frac{1}{2} t\right)^{2}-2 l t \geq 0$, that is,

$$
f(t) \equiv(t-2 l(2-M+2 \sqrt{1-M}))(t-2 l(2-M-2 \sqrt{1-M})) \geq 0 .
$$

We are interested in the cases where $M>\frac{1}{2 l}\left(\Delta_{y}-l \Delta_{x}\right)$ (because $p^{\text {all }}$ is certainly optimal otherwise), that is, $t<2 l M$. The function $f(t)$ satisfies $f(0)>0$. Moreover, $f(2 l M)<0$. Therefore, $f(t) \geq 0$ if and only if $t$ is lower than the first root, that is, $\Delta_{y}-l \Delta_{x} \leq$ $2 l(2-M-2 \sqrt{1-M})$, or

$$
\Delta_{y} \leq l \Delta_{x}+2 l(2-M-2 \sqrt{1-M})=\lg \left(\Delta_{x}\right) .
$$

We note that the change in the definition of the function $g\left(\Delta_{x}\right)$ happens at the point where $M=\frac{1}{2 l}\left(\Delta_{y}+3 l \Delta_{x}\right)$ (which separates the regions where either $p^{\text {int }}$ or $p^{\max }$ are candidates). Then, the value $\Delta_{x}$ where the change happens is the solution of the following system of equations (in $\Delta_{x}$ and $\Delta_{h}$ ):

$$
\begin{aligned}
M & =\frac{1}{2 l}\left(\Delta_{y}+3 l \Delta_{x}\right) \\
\Delta_{y} & =l \Delta_{x}+2 l(2-M-2 \sqrt{1-M})
\end{aligned}
$$

that is,

$$
\Delta_{x}=\sqrt{1-M}-(1-M)
$$


which is a positive value. The function $g\left(\Delta_{x}\right)$, as it is defined in the main text just before Proposition 1, is continuous because it is continuous at the point $\sqrt{1-M}-(1-M)$, and it is also continuously differentiable.

Once we have analyzed the optimal price in each of the three zones, it easily follows that the solution is continuous, in the sense that if the optimal price is $p^{\text {all }}$ (resp. $p^{\max }$ ) in the second zone, then it is also optimal if we decrease $\Delta_{c}$ and enter the third zone. Therefore, the optimal firm 1's pricing policy is the one described in the proposition.

Finally, it is easy to check that the profits are continuous: (a) over the line $\Delta_{y}=$ $2 l M-3 l \Delta_{x}$, we have $\Pi^{1}\left(p^{\max }\right)=\Pi^{1}\left(p^{\text {int }}\right)$. (b) If the condition $\Delta_{y}=l g\left(\Delta_{x}\right)$ holds we have two cases: (b.1) For $g\left(\Delta_{x}\right) \equiv \frac{\left(1+M-\Delta_{x}\right)}{\left(1-M+\Delta_{x}\right)} \Delta_{x}, \Pi^{1}\left(p^{\text {all }}\right)=\Pi^{1}\left(p^{\max }\right)$ and (b.2) for $g\left(\Delta_{x}\right) \equiv \Delta_{x}+2(2-M-2 \sqrt{1-M})$, then $\Pi^{1}\left(p^{\text {all }}\right)=\Pi^{1}\left(p^{\text {int }}\right)$.

Proof of Corollary 1. We denote $\Pi^{1}\left(\Delta_{x}, \Delta_{y}\right)$ the firm 1's profits as a function of $\Delta_{x}$ and $\Delta_{y}$.

In Region b.i, where $p^{1 *}=p^{\max }$ :

$\Pi_{\Delta_{y}}^{1}\left(\Delta_{x}, \Delta_{y}\right)=b\left(M-\Delta_{x}\right)>0 ; \quad \Pi_{\Delta_{y} \Delta_{y}}^{1}\left(\Delta_{x}, \Delta_{y}\right)=0$.

$\Pi_{\Delta_{x}}^{1}\left(\Delta_{x}, \Delta_{y}\right)=b\left(l M-\left(2 l \Delta_{x}+\Delta_{y}\right)\right)$, which is positive for $\Delta_{y}=-l \Delta_{x}$ and negative for $\Delta_{y}=2 l M-3 l \Delta_{x} ; \quad \Pi_{\Delta_{x} \Delta_{x}}^{1}\left(\Delta_{x}, \Delta_{y}\right)=-2 b l<0$.

In Region b.ii, where $p^{1 *}=p^{\text {int }}$ :

$$
\begin{aligned}
& \Pi_{\Delta_{y}}^{1}\left(\Delta_{x}, \Delta_{y}\right)=\frac{b}{4 l}\left(2 l M+\Delta_{y}-l \Delta_{x}\right)>0 ; \quad \Pi_{\Delta_{y} \Delta_{y}}^{1}\left(\Delta_{x}, \Delta_{y}\right)=\frac{b l}{4}>0 . \\
& \Pi_{\Delta_{x}}^{1}\left(\Delta_{x}, \Delta_{y}\right)=-\frac{b}{4}\left(2 l M+\Delta_{y}-l \Delta_{x}\right)<0 ; \quad \Pi_{\Delta_{x} \Delta_{x}}^{1}\left(\Delta_{x}, \Delta_{y}\right)=\frac{b l}{4}>0 .
\end{aligned}
$$

In Region $\mathrm{c}$, where $p^{1 *}=p^{\text {all }}$ :

$$
\begin{aligned}
& \Pi_{\Delta_{y}}^{1}\left(\Delta_{x}, \Delta_{y}\right)=b>0 ; \quad \Pi_{\Delta_{y} \Delta_{y}}^{1}\left(\Delta_{x}, \Delta_{y}\right)=0 . \\
& \Pi_{\Delta_{x}}^{1}\left(\Delta_{x}, \Delta_{y}\right)=-l b<0 ; \quad \Pi_{\Delta_{x} \Delta_{x}}^{1}\left(\Delta_{x}, \Delta_{y}\right)=0 .
\end{aligned}
$$

Proof of Proposition 2. Proofs for Region a and Region b.i are immediate.

In Region b.ii the variation in health system surplus, that is, (in case $\left.x^{1} \geq x^{0}\right)^{29}$ the difference for patients in $[\tilde{x}, 1]$ from being treated with drug $\left(h^{0}, x^{0}\right)$ at price $c^{0}$ and being treated with drug $\left(h^{1}, x^{1}\right)$ at price $p^{i n t}$, is the sum of the area defined by a triangle for $\left[\tilde{x}, x^{1}\right]$ and the area defined by a rhomboid for $\left[x^{1}, 1\right]$. For the patients in $\left[\tilde{x}, x^{1}\right]$, $\Delta C S=\frac{1}{2}\left(x^{1}-\tilde{x}\left(p^{i n t}\right)\right)\left(b h^{1}-p^{i n t}-b\left(h^{0}-l\left(x^{1}-x^{0}\right)\right)+c^{0}\right)$. Substituting $\tilde{x}\left(p^{i n t}\right)$ and $p^{\text {int }}$, we have

$$
\Delta C S=\frac{b}{16 l}\left(\Delta_{y}+3 l \Delta_{x}-2 l M\right) .
$$

For the patients in $\left[x^{1}, 1\right], \Delta C S=\left(1-x^{1}\right)\left(b h^{1}-p^{i n t}-b\left(h^{0}-l\left(x^{1}-x^{0}\right)\right)+c^{0}\right)$. Substituting $p^{i n t}$ and using $\left(1-x^{1}\right)=\left(M-\Delta_{x}\right)$, we have

$$
\Delta C S=\frac{b}{2}\left(M-\Delta_{x}\right)\left(\Delta_{y}+3 l \Delta_{x}-2 l M\right) .
$$

\footnotetext{
${ }^{29}$ The case $x^{1}<x^{0}$ is similar.
} 
Hence, adding both intervals, we obtain

$$
\Delta C S\left(p^{i n t}\right)=\frac{b}{16 l}\left(\Delta_{y}+3 l \Delta_{x}-2 l M\right)\left(\Delta_{y}-5 l \Delta_{x}+6 l M\right) .
$$

In Region c the variation in the health system surplus is similar to the one previously analyzed, but taking into account that for $p^{\text {all }}$ (considering again that $x^{1}>x^{0}$ ) patients in $\left[0, x^{0}\right]$ have the same surplus as without the new drug. For the patients in $\left[x^{0}, x^{1}\right]$,

$$
\Delta C S=\frac{1}{2}\left(x^{1}-x^{0}\right)\left(b h^{1}-p^{a l l}-b\left(h^{0}-l\left(x^{1}-x^{0}\right)\right)+c^{0}\right) .
$$

Substituting $p^{\text {all }}$ we have

$$
\Delta C S=b l \Delta_{x}^{2}
$$

For $\left[x^{1}, 1\right], \Delta C S=\left(1-x^{1}\right)\left(b h^{1}-p^{a l l}-b\left(h^{0}-l\left(x^{1}-x^{0}\right)\right)+c^{0}\right)$. Substituting $p^{\text {all }}$ and using $\left(1-x^{1}\right)=\left(M-\Delta_{x}\right)$,we obtain

$$
\Delta C S=2 b l \Delta_{x}\left(M-\Delta_{x}\right)
$$

Adding the two parts, we have

$$
\Delta C S\left(p^{a l l}\right)=b l \Delta_{x}\left(2 M-\Delta_{x}\right) .
$$

Proof of Corollary 2. We denote $\Delta C S\left(\Delta_{x}, \Delta_{y}\right)$ the variation in consumer surplus as a function of $\Delta_{x}$ and $\Delta_{y}$.

In Region a and Region b.i all the derivatives are zero.

In Region b.ii:

$\Delta C S_{\Delta_{y}}\left(\Delta_{x}, \Delta_{y}\right)=\frac{b}{8 l}\left(\Delta_{y}-l \Delta_{x}+2 l M\right)>\frac{b}{8 l}\left(-2 l \Delta_{x}+2 M l\right)>0$.

$\Delta C S_{\Delta_{y} \Delta_{y}}\left(\Delta_{x}, \Delta_{y}\right)=\frac{b}{8 l}>0$.

$\Delta C S_{\Delta_{x}}\left(\Delta_{x}, \Delta_{y}\right)=\frac{1}{8}\left(-\Delta_{y}-15 l \Delta_{x}+14 l M\right)$. We note that, at $M=\frac{1}{2 l}\left(\Delta_{y}+3 l \Delta_{x}\right)$, $\Delta C S_{\Delta_{x}}\left(\Delta_{x}, \Delta_{y}\right)=\frac{3}{4}\left(\Delta_{y}+l \Delta_{x}\right)>0$ because $\Delta_{y}>-l \Delta_{x}$. Moreover, $\Delta C S_{\Delta_{x}}\left(\Delta_{x}, \Delta_{y}\right)<$ $\frac{1}{8}\left(-14 l \Delta_{x}+14 l M\right)$. Hence, $\Delta C S_{\Delta_{x}}\left(\Delta_{x}=M, \Delta_{y}\right)<0$.

$\Delta C S_{\Delta_{x} \Delta_{x}}\left(\Delta_{x}, \Delta_{y}\right)=-\frac{15}{8} b l<0$

In Region c:

$$
\begin{aligned}
& \Delta C S_{\Delta_{y}}\left(\Delta_{x}, \Delta_{y}\right)=0 ; \Delta C S_{\Delta_{y} \Delta_{y}}\left(\Delta_{x}, \Delta_{y}\right)=0 . \\
& \Delta C S_{\Delta_{x}}\left(\Delta_{x}, \Delta_{y}\right)=2 b l\left(M-\Delta_{x}\right)>0 . \\
& \Delta C S_{\Delta_{x} \Delta_{x}}\left(\Delta_{x}, \Delta_{y}\right)=-2 b l<0 .
\end{aligned}
$$

Finally, the behavior of $\triangle C S$ at the borders between regions follows from the behavior of the optimal price $p^{1 *}$ that we discussed following Proposition 1.

Proof of Proposition 3. In case of a successful project, the new drug $\left(x=x^{0}, y\right)$ lies either in Region a (for $y \leq y^{0}$ ) or Region $\mathrm{c}$ with $x=x^{0}$ (for $y>y^{0}$ ). In the first 
case (see Proposition 2), $\Delta C S\left(x=x^{0}, y\right)=\Delta C S\left(c^{1}\right)=0$. In the second case, $\Delta C S(x=$ $\left.x^{0}, y\right)=\Delta C S\left(p^{\text {all }}\right)=b l \Delta_{x}\left(2 M-\Delta_{x}\right)=0$ because $\Delta_{x}=0$. Therefore, $\Pi_{i n}(I)=W_{i n}(I)$ for all $I$ and the optimal investment levels for the two functions coincide.

The argument for a horizontal incremental innovation process is similar because the new drug would lie in Region b.i where $\Delta C S\left(x, y=y^{0}\right)=\Delta C S\left(p^{\max }\right)=0$.

Proof of Proposition 4. There is some positive probability that the new drug $\left(x^{1}, y^{1}\right)$ lies in Region $\mathrm{c}$ with $x^{1} \neq x^{0}$. Depending on the level of $y^{1}$, there can also be a positive probability that the new drug lies in Region b.ii). In both regions, $\Delta C S\left(x, y^{1}\right)>$ 0 . Given that $\Delta C S(x, y) \geq 0$ for every new drug, we have $\frac{d \Pi_{r a}(I)}{d I}<\frac{d W_{r a}(I)}{d I}$ for every $I$, which implies that $I_{r a}^{1}<I_{r a}^{*}$.

Proof of Proposition 5. Denoting by $I_{Z}^{1}$ and $I_{Z}^{*}$ for $Z=i n$, $r a$ the optimal level of investment in a process of type $Z$ for firm 1 and the social planner, respectively, then

$$
\begin{aligned}
& \Pi_{i n}\left(I_{i n}^{1}\right)=W_{i n}\left(I_{i n}^{*}\right) \\
& \Pi_{r a}\left(I_{r a}^{1}\right)<W_{r a}\left(I_{r a}^{*}\right) .
\end{aligned}
$$

It may happen that

$$
W_{r a}\left(I_{r a}^{*}\right)>W_{i n}\left(I_{i n}^{*}\right)=\Pi_{i n}\left(I_{i n}^{1}\right)>\Pi_{r a}\left(I_{r a}^{1}\right)
$$

but it can never be the case that

$$
W_{r a}\left(I_{r a}^{*}\right)<W_{i n}\left(I_{i n}^{*}\right)=\Pi_{i n}\left(I_{i n}^{1}\right)<\Pi_{r a}\left(I_{r a}^{1}\right),
$$

which implies the result. 